\title{
Anti-tumor effect of aloe-emodin on cervical cancer cells was associated with human papillomavirus E6/E7 and glucose metabolism
}

This article was published in the following Dove Press journal:

OncoTargets and Therapy

\author{
Rui Gao ${ }^{1,2}$ \\ Xiaowen $\mathrm{Wu}^{1,3}$ \\ Zhi Huang ${ }^{1,3}$ \\ Bi Wang ${ }^{1,4}$ \\ Fenghu $\mathrm{Li}^{5}$ \\ Hui Xu ${ }^{1,4}$ \\ Li $\operatorname{Ran}^{5}$
}

'Key Laboratory of Endemic and Ethnic Diseases, The Key Laboratory of Medical Molecular Biology in Guizhou Medical University, Guiyang, 550002, People's Republic of China; ${ }^{2}$ Guizhou International Travel Healthcare Center, Guiyang 550005, People's Republic of China; ${ }^{3}$ School of Medical Imaging of Guizhou Medical University, Guiyang 550002, People's Republic of China; ${ }^{4}$ Department of Paediatrics, Maternal and Child Health Hospital of Guiyang City, Guiyang 550003, People's Republic of China; ${ }^{5}$ Department of Breast and Gynecologic Oncology, The Affiliated Cancer Hospital of Guizhou Medical University, Guiyang 550004, People's Republic of China

Correspondence: Hui Xu

Key Laboratory of Endemic and Ethnic

Diseases, The Key Laboratory of Medical

Molecular Biology in Guizhou Medical

University, No. 63, Ruijin South Road

Nanming District, Guiyang 550002,

People's Republic of China

Tel +8608518687398I

Email jadenxu@sohu.com

Li Ran

Department of Breast and Gynecologic Oncology, The Affiliated Cancer Hospital of Guizhou Medical University, No. I, Beijinxi Road, Yunyan District, Guiyang 550004, People's Republic of China

$\mathrm{Tel}+86085186855119$

Email ranlil7I@hotmail.com
Background: Aloe-emodin, an anthraquinone present in aloe latex, has been shown to have anti-proliferative properties in cervical cancer disease, all cases of which are almost caused by human papillomavirus (HPV), with the products of E6/E7. It is suggested that aloeemodin may play an important role in HPV-induced cervical cancer cells.

Methods: Hela and SiHa cells were treated with various concentrations of aloe-emodin. MTT assay and flow cytometry were used to identify the cell growth and apoptosis. The expressions of HPV E6, E7 and GLUT1 (glucose transporter-1) were detected by real-time quantitative polymerase chain reaction (qRT-PCR) and Western blot (WB). The glucose uptake, lactate production and ATP production in HeLa and SiHa cells were also investigated.

Result: The results indicate that aloe-emodin promoted the apoptosis of $\mathrm{HeLa}$ and $\mathrm{SiHa}$ cells and decreased the expressions of HPV-related protein E6 and E7. Furthermore, aloe-emodin inhibited glucose metabolism by reducing GLUT1 expression. Overexpression of GLUT1 significantly weakened the apoptosis induced by aloe-emodin in HeLa cells.

Conclusion: In this study, we found that aloe-emodin induce apoptosis of cervical cancer cells, which was associated with HPV E6 and E7 and glucose metabolism.

Keywords: cervical cancer, aloe-emodin, E6/E7, glucose metabolism

\section{Introduction}

Aloe-emodin (1,8-dihydroxy-3-hydroxymethyl-9,10-anthracenedione, AE), a natural compound from the root and rhizome of Rheum palmatum, has an antiproliferative effect on different human cancer cell lines. According to previous reports, AE can inhibit invasion of nasopharyngeal carcinoma cells, ${ }^{1}$ human oral cancer KB cells and hepatocarcinoma cells. ${ }^{2,3}$ In addition, AE was also worked as a growth inhibitor on cervical cancer cells. ${ }^{4}$

Cervical cancer is the most common gynecological malignancies, the incidence of which is the highest in malignant tumor in women and only second to breast cancer worldwide. ${ }^{5,6}$ Nowadays, the therapies of cervical cancer are mainly surgical treatment, radiotherapy, chemotherapy and other combined treatments. ${ }^{7}$ Just as other malignant tumors, the factors that affect the survival of patients include poor prognosis, tumor invasion and metastasis in advanced stage. ${ }^{6}$ Therefore, it is of great importance to explore the effective method for the diagnosis and treatment of cervical cancer. 
Evidences show that human papillomaviruses (HPVs) infection, the association of which with human cancer was first proposed more than three decades ago by Harald zurHausen, ${ }^{8}$ is virtually present in all cases of cervical cancer. HPV16 and HPV18 belong to high-risk HPV virus and are the most frequently found types in cervical cancers around the world, accounting for approximately $50 \%$ and $20 \%$ of the cases, respectively. The products of the early genes in the high-risk mucosal HPV types, E6 and E7, play a key role in HPV-associated cancers. ${ }^{9}$ Studies have found that there are sustained expressions of viral E6 and E7 proteins in HPV-associated lesions and cancers, which was considered to be the major inducer of cell transformation. ${ }^{10,11}$

Glucose metabolism provides energy to the process of tumor cells growth. ${ }^{12}$ Glucose transporter proteins (GLUTs) which mediated glucose uptake is the main carrier. Facilitative glucose transporters transport glucose through the hydrophobic cell membrane along the concentration gradient without energy consumption. ${ }^{13}$ So far there have been eight kinds of glucose transporter protein (glucouse transporters) identified (Glut1-5 and Glut7-9). GLUT1 is present at variable levels in many tissues and is believed to be responsible for basal glucose uptake. ${ }^{14}$ Increased GLUT1 expression has been discovered in many cancers, including cervical carcinoma, cervical cancer, lung cancer and colorectal carcinoma. ${ }^{15-18}$

In this study, in order to explore the potential mechanism of $\mathrm{AE}$ on cervical cancer, we investigated the effects of $\mathrm{AE}$ on the expressions of HPV E6/E7 and glucose metabolism in $\mathrm{HeLa}$ as well as in SiHa cells, finding that AE may promote apoptosis of cervical cancer cells by inhibiting the expressions of HPV E6/E7 and regulating glucose metabolism.

\section{Materials and methods}

\section{Cell culture and drug treatment}

The human cervical carcinoma cell lines Hela and $\mathrm{SiHa}$ were purchased from the Cell Bank of the Chinese Academy of Sciences (Shanghai, China). All cells were cultured in Dulbecco's modified Eagle's medium (DMEM, Gibco, Carlsbad, CA, USA) supplement with $10 \%$ fetal bovine serum (FBS, Invitrogen, Carlsbad, CA, USA) and 1\% penicillin-streptomycin at $37^{\circ} \mathrm{C}$ with $5 \% \mathrm{CO}_{2} .0 .1 \mathrm{M} \mathrm{AE}$ stock (Sigma) dissolved in dimethyl sulfoxide (DMSO) was diluted into working concentrations by DMEM medium to stimulate cells. The negative control was added with the same volume of DMSO in the culture medium.

\section{Cell transfection}

The overexpression of GLUT1 was conducted in accordance with the protocol described previously. ${ }^{19}$ Briefly, when Hela cells in 6-well plates grew to $60-70 \%$ confluence, $4 \mu \mathrm{g}$ empty pcDNA3.1 vectors or $4 \mu \mathrm{g}$ pcDNA3.1-GLUT1 were transfected into cells with Lipofectamine 2000 reagent (Invitrogen) in serum-free medium, which was replaced by complete medium containing 10\% FBS 5 hrs later. After 2 days, the cells were treated with $\mathrm{AE}$ as above mentioned.

\section{MTT assay}

MTT [3-(4,5-dimethylthiazol-2-yl)-2,5-diphenyl-2Htetrazolium bromide] assay was used to measure viable cell growth. $1 \times 10^{5}$ cells/well were seeded in 96-well plates followed by treating with $\mathrm{AE}$ at different doses $(10,20,50,100$, $200 \mu \mathrm{M})$ for $24 \mathrm{hrs}$. Then, the cells were incubated with the MTT solution for $4 \mathrm{hrs}$ which was dissolved by formazan. Finally, the optical density (OD) values were detected at $550 \mathrm{~nm}$ with a Wellscan reader (Labsystems, Santa Fe, NM, USA). The cell proliferation rate was calculated as follows: $\left(\mathrm{OD}_{\text {experiment }} / \mathrm{OD}_{\text {control }}\right) \times 100 \%{ }^{20}$

\section{Cell apoptosis assay by flow cytometry}

$4 \times 10^{5}$ Hela and $\mathrm{SiHa}$ cells per well were maintained in mediums overnight for adherence and then administrated with $\mathrm{AE}$ $(50 \mu \mathrm{M}, 100 \mu \mathrm{M})$ for $24 \mathrm{hrs}$. Morphological characteristics of the cells were observed with an inverted microscope. Flow cytometry assay was carried out with an Annexin V-FITC/PI apoptosis detection kit (BD Biosciences) following the manufacturer's instructions.

\section{Western blotting}

$2 \times 10^{5}$ cells were lysed with immune-precipitation buffer on ice and centrifugated at $13,000 \times \mathrm{g}$ for $15 \mathrm{~min}$ at $4^{\circ} \mathrm{C}$ to collect the supernatant. The lysates were boiled and $40 \mu \mathrm{g}$ total cell lysates were separated by $10 \%$ sodium dodecyl sulfate polyacrylamide gel electrophoresis (SDS-PAGE), which was transferred onto a polyvinylidene fluoride membrane (Millipore, Bedford, MA, USA). The protein bands were detected by incubating with antibodies including anti-HPV 16/18 E6, E7, GLUT1 and glyceraldehyde-3-phosphate dehydrogenase (GAPDH), from Boster Bioengineering (Wuhan, China). Enhanced chemiluminescence reagents (Beyotime) was used to visualize the proteins and image $\mathrm{J}$ software was applied to measure the relative protein expressions. 


\section{Reverse transcription-quantitative PCR (qRT-PCR)}

Two micrograms of total RNA, which was extracted from transfected cells using TRIzol reagent (Life Technologies, Carlsbad, CA, USA), was reversed to cDNA with the HighCapacity cDNA Archive Kit (Applied Biosystems, Foster City, CA, USA). Real-time PCR was performed with Maxima SYBR Green/ROX qPCR Master Mix (Thermo Scientific, CA, USA) to analyze the gene expression levels and cervical cancer cells replication rate. GAPDH gene was used as an endogenous control. The relative expression values were measured with the $2-\Delta \Delta \mathrm{Ct}$ method. The primer sequences were described in Table 1.

\section{Glucose metabolism analysis including glucose uptake, lactate and ATP production}

$\mathrm{HeLa}$ and $\mathrm{SiHa}$ cells were starved overnight in serum-free medium and then treated with $\mathrm{AE}$ at concentrations of 50 and $100 \mu \mathrm{M}$ for $0,4,8,12$ and $24 \mathrm{~h}$. According to the manufacturers' instructions, glucose uptake assay was performed with a glucose uptake assay kit (ab136955, Abcam). Lactate production was analyzed using an L-Lactate assay kit (ab65331, Abcam). The measurement of ATP was carried out with an ATP assay kit (ab83355, Abcam). The relative glucose uptake, lactate production and ATP production of AE groups were calculated with the percentage of the DMSO group.

\section{Statistical analysis}

All the data were shown as means \pm SD. The statistical analysis was performed with the SPSS 12.0 software. A value of $p<0.05$ was considered to be statistically significant.

\section{Results}

\section{Effect of $A E$ on cell growth in cervical cancer cells}

MTT assay was performed to evaluate the effect of AE on cell growth. Hela and $\mathrm{SiHa}$ cells were treated with $\mathrm{AE}$ of concentrations from 10 to $200 \mu \mathrm{M}$. As shown in Figure $1 \mathrm{~A}, \mathrm{AE}$ obviously inhibited the growth of cells in a concentrationdependent manner. To evaluate the effect of AE on cells' apoptosis, Hela and $\mathrm{SiHa}$ cells were treated with $\mathrm{AE}$ at concentrations of 50 and $100 \mu \mathrm{M}$ for $24 \mathrm{~h}$, displaying that $\mathrm{AE}$ remarkedly increased the apoptosis rates in Hela and SiHa cells (Figure 1B).

\section{Effects of $A E$ on the expression of HPV-I6/I8 E6 and E7}

qRT-PCR and WB analysis were used to identify the mRNA and protein levels of HPV-16/18 E6 and E7 in Hela and SiHa cells after treatment of AE. It was shown that the mRNA levels of HPV18-E6/E7 and HPV16-E6/E7 are inhibited in a concentration-dependent manner in Hela cells (Figure 2A) and in SiHa cells (Figure 2B), respectively. Accordingly, similar results were revealed at protein levels. As shown in Figure 2C-D, protein levels of E6 and E7 were significantly down-regulated in Hela cells and $\mathrm{SiHa}$ cells with corresponding quantitative analysis in histogram.

\section{Effect of $A E$ on glucose metabolism in cervical cancer cells}

Malignant cells are known to accelerate metabolism, high glucose requirements, and increased glucose uptake. To evaluate the effect of AE on cell metabolism, glucose uptake, lactate production and ATP production were measured. Hela

Table I Sequences of primers for quantitative real-time polymerase chain reaction

\begin{tabular}{|l|l|l|}
\hline Primer & & Sequences \\
\hline HPVI6-E6 & Forward & 5'-CTTACCACAGTTATGCACAGAG-3' \\
& Reverse & 5'-CTACGTGTTCTTGATGATCTG-3' \\
HPVI6-E7 & Forward & 5'-GGAGGATGAAATAGATGGTC-3' \\
& Reverse & 5'-GTCTTCCAAAGTACGAATGTC-3' \\
HPVI8-E6 & Forward & 5'-GACCCTACAAGCTACCTGATC-3' \\
& Reverse & 5'-GGATTCAACGGTTTCTCTGG-3' \\
HPVI8-E7 & Forward & 5'-CTCAGAGGAAGAAAACGATG-3' \\
& Reverse & 5'-CACAAAGGACAGGGTGTTC-3' \\
GLUTI & Forward & 5'-CCGCGAATTCATGGATCCCAGCAGCAAGAAG-3' \\
GAPDH & Reverse & 5'-CCGCGGATCCTCACACTTGGGAGTCCGCCCC-3' \\
& Forward & 5'-GGAGCGAGATCCCTCCAAAAT-3' \\
& Reverse & 5'-GGCTGTTGTCATACTTCTCATGG-3' \\
\hline
\end{tabular}



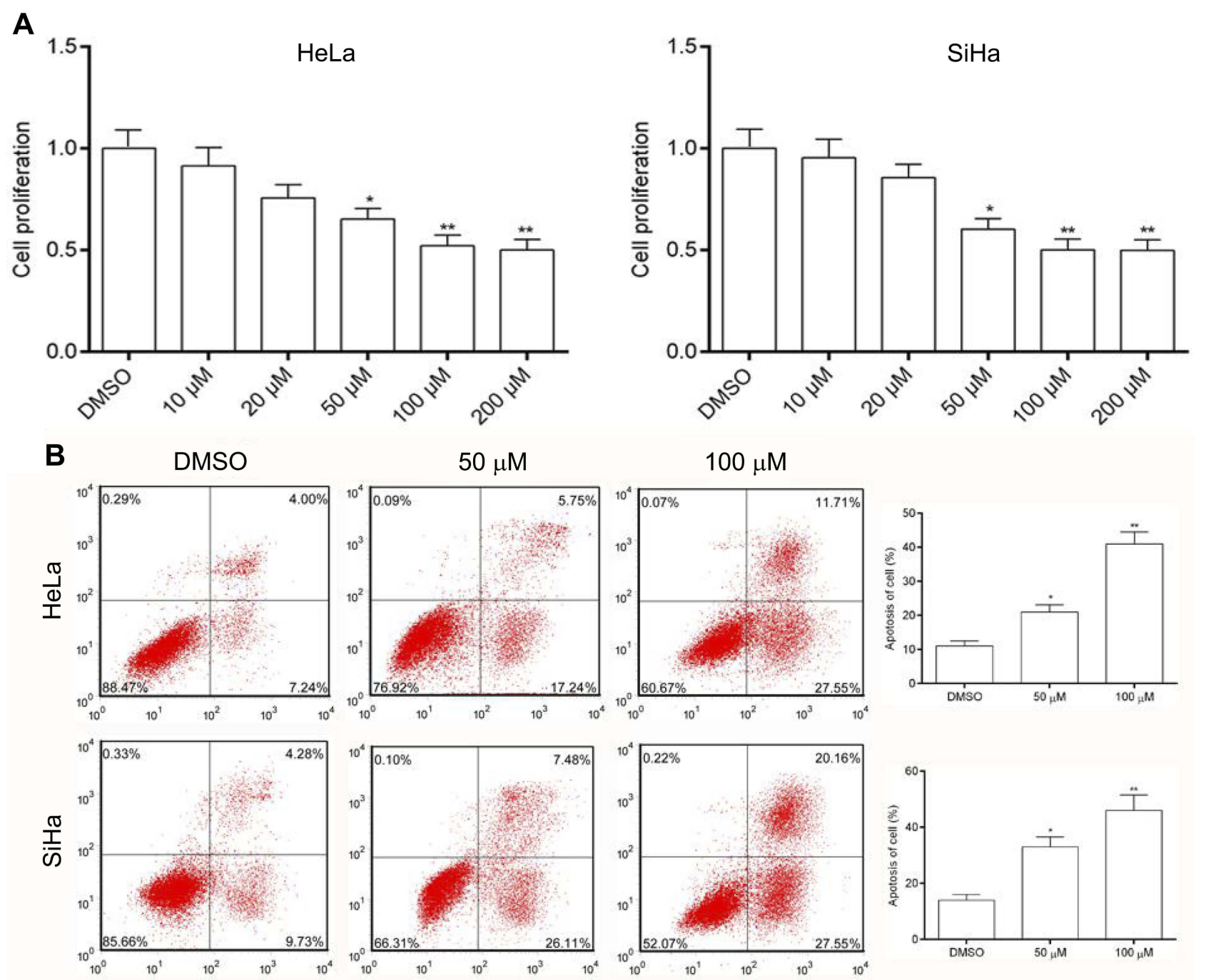

Figure I Effects of $A E$ on cell growth in cervical cancer cells. (A) Anti-proliferative effects of $A E$ in Hela and SiHa cells. Cells were treated with various concentrations of $A E$ and the proliferation measured using the MTT assay. (B) Flow cytometry assay was used to measure the apoptosis rate after treated with $A E$. $* p<0.05$ and $* * p<0.0$ I compared with the data from the respective control group detected at same time point.

and siHa cells were treated with AE at concentrations of 50 and $100 \mu \mathrm{M}$, showing that $\mathrm{AE}$ inhibits metabolism by controlling glucose uptake (Figure 3A), lactate production (Figure 3B) and ATP production (Figure $3 \mathrm{C}$ ) in a concentration and timedependent manner.

\section{AE inhibited glucose metabolism via decreasing GLUTI expression in cervical cancer cells}

Glucose transportion across mammalian cytoplasmic membrane, which is mediated by GLUTs, is the first rate-limiting step in glucose metabolism. Therefore, we hypothesized that the inhibitory effect of AE on tumor metabolism was achieved by adjusting the level of GLUTs. As expected, AE obviously decreased the mRNA level of GLUT1 in HeLa and SiHa cells (Figure 4A-B). Furthermore, the protein expression of GLUT1 was also significantly depressed by AE (Figure 4C-D). However, when GLUT1 was overexpressed in HeLa cells, the inhibitory effects of AE on glucose uptake, lactic production and ATP production were markedly weakened (Figure 5B-D). These results indicated that $\mathrm{AE}$ inhibited glucose metabolism via decreasing GLUT1 expression. The transfection efficiency of pcDNA3.1-GLUT1 was evaluated by WB. As shown in Figure 5A, HeLa cells transfected with pcDNA3.1-GLUT1 


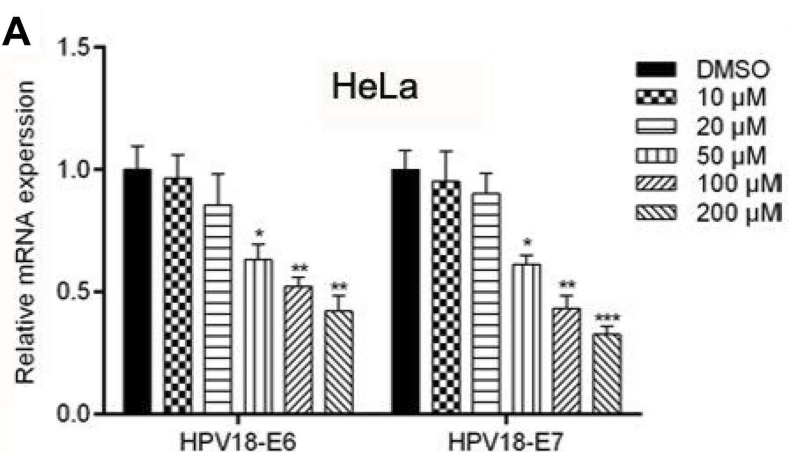

C
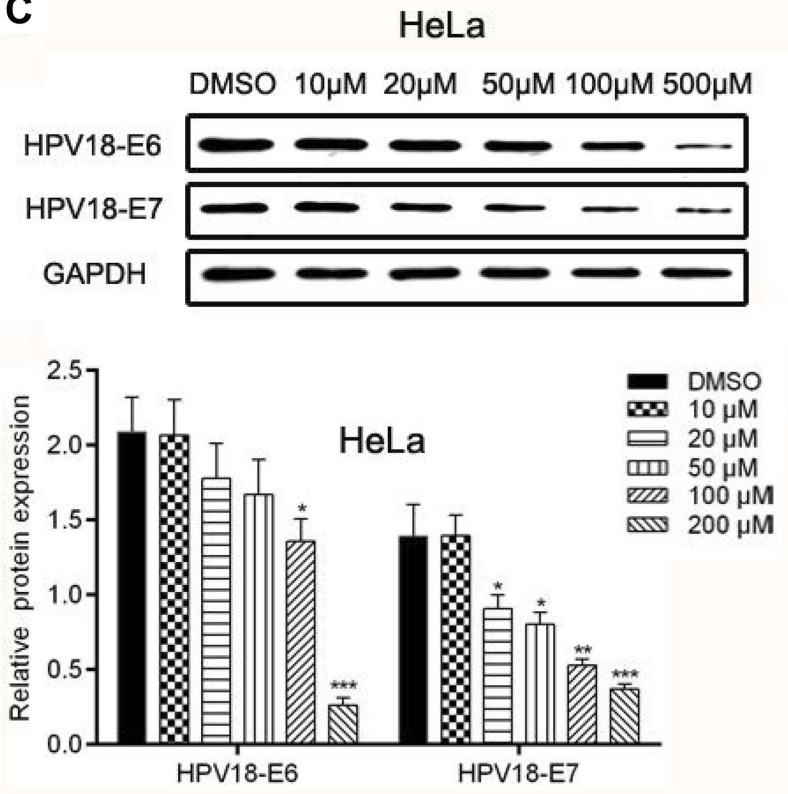

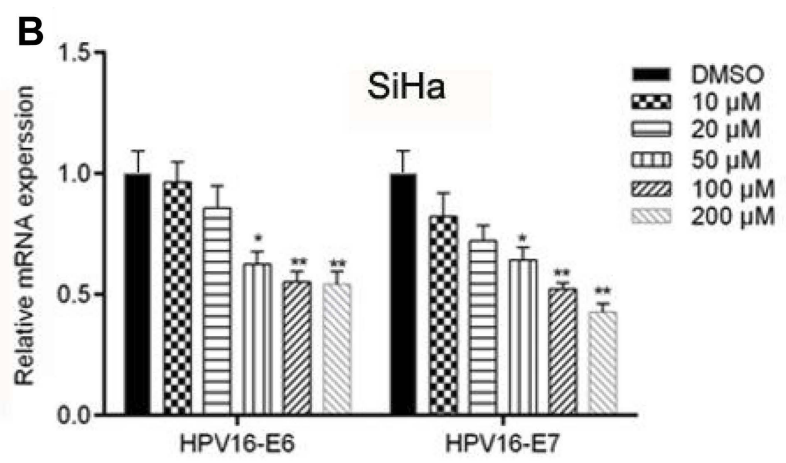

D
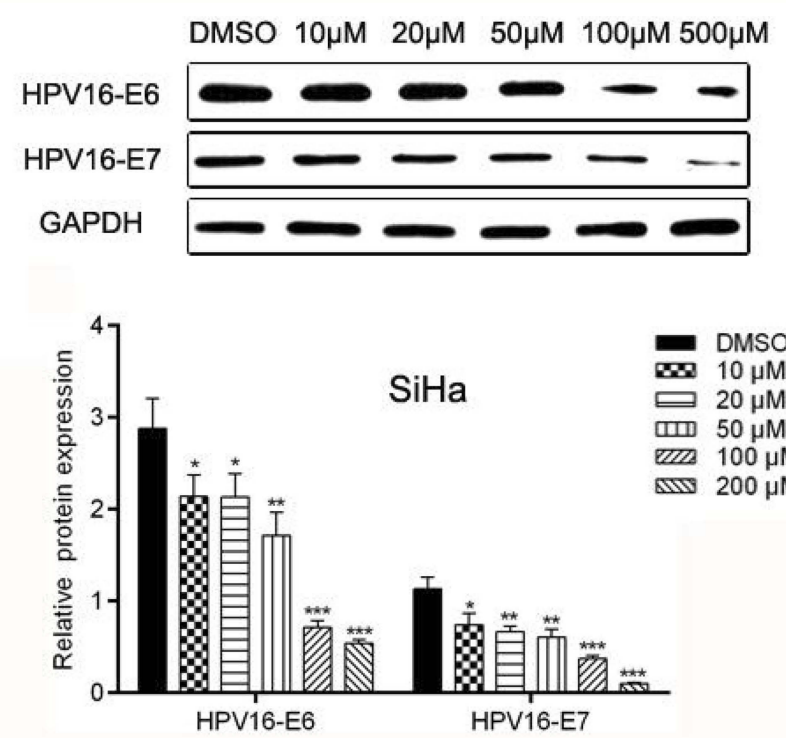

Figure 2 Effects of AE on the expression of HPV-associated genes and proteins. (A-B) The expression of E6 and E7 mRNA were determined using qPCR. (C-D) The protein expression levels of E6 and E7 were determined by WB. $* p<0.05, * * p<0.01$ and $* * * p<0.001$ compared with the data from the respective control group detected at same time point.

displayed a much higher GLUT1 level than the cells transfected with pcDNA3.1 vectors.

\section{Overexpression of GLUTI inhibited the AE-induced apoptosis of cervical cancer cells}

MTT assay (Figure 6A) showed that under AE stress, overexpression of GLUT1 significantly promoted the proliferation of HeLa cells compared to the cells transfected with pcDNA3.1 vectors. Flow cytometry assay (Figure 6B and C) displayed that overexpression of GLUT1 obviously decreased the apoptosis induced by AE.

\section{Discussion}

As a traditional Oriental herb, it has been found that AE has anticancer functions in several cancer cell lines. ${ }^{21,22}$
More importantly, no obvious toxicity of AE was observed in vivo. ${ }^{21}$ However, the anticancer molecular mechanisms of AE are still unclear, especially for cervical cancer cells.

High-risk human papillomavirus (hrHPV) has been identified as a key factor in the development of cervical cancer. ${ }^{23}$ $\mathrm{HPV}$-associated cancers are closely related to the persistence of HPV and the accumulation of chromosomal recombination. ${ }^{9}$ Among the early genes, E6 and E7 are the two most famous oncogenic proteins which are consistently expressed in HPV-associated malignancies and are responsible for the proliferation of cancer cells. Evidence has shown that the expression of E6 and E7 during natural HPV infection could immortalize cells efficiently. However, inhibition of E6 and E7 expression could block the malignant phenotype of cervical cancer cells. ${ }^{7}$ In the present study, we detected that AE inhibited the proliferation and promoted the apoptosis of Hela and SiHa cells, which was consistent with others' research. To 

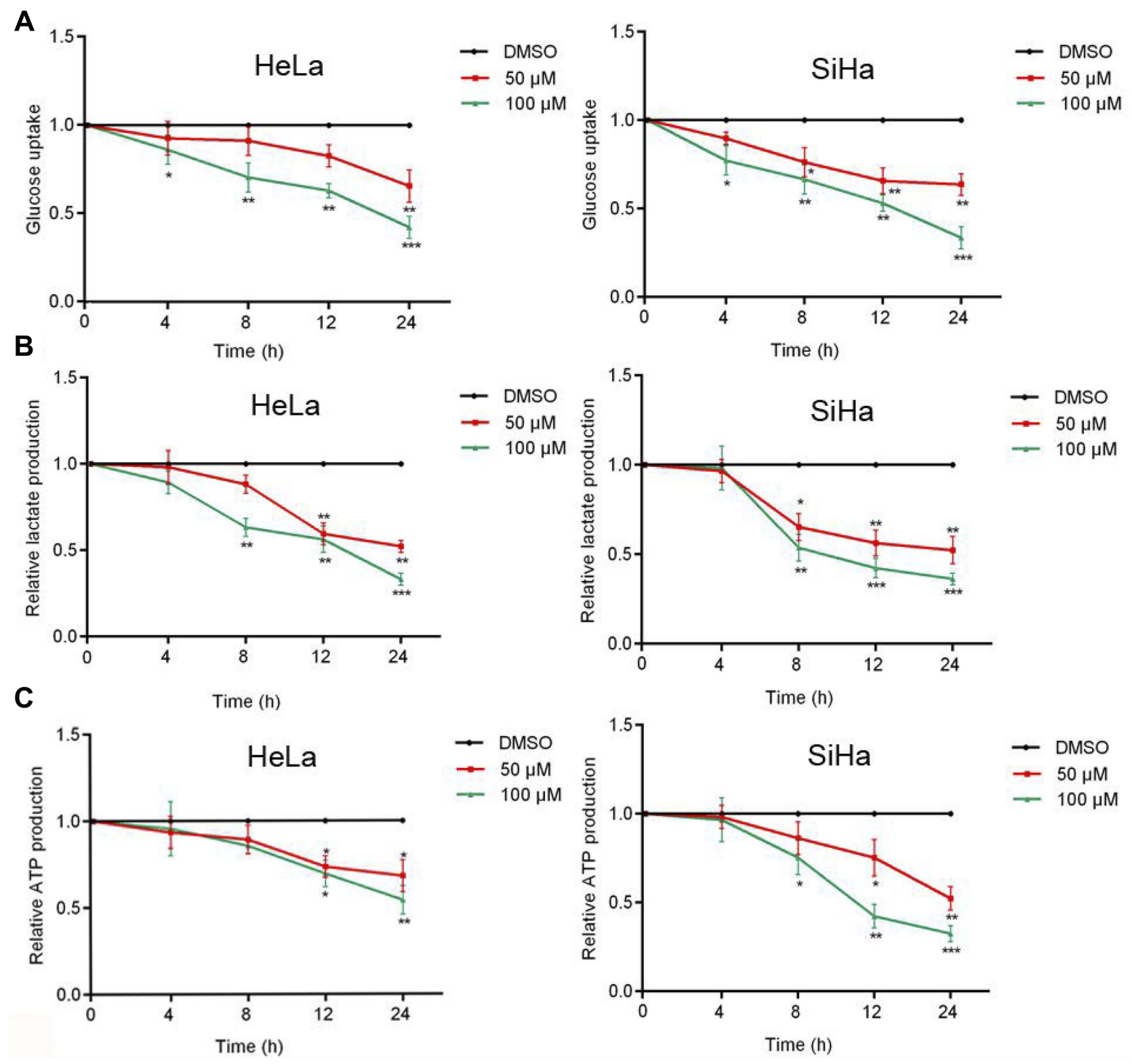

Figure 3 Effect of AE on glucose metabolism. (A) After treated with AE at a concentration of $50 \mu \mathrm{ml} / \mathrm{L}$ and $100 \mu \mathrm{ml} / \mathrm{L}$, the glucose uptake of Hela and SiHa cells decreased. (B) Similarly, lactate production decreased after treated with AE in Hela and SiHa cells. (C) The yield of ATP decreased with the decrease of glucose uptake and lactate production. Glucose metabolism is suppressed in tumor cells. ${ }^{*} p<0.05, * * p<0.0$ I and ${ }^{* * *} p<0.00$ I compared with the data from the respective control group detected at same time point.

examine the mechanism responsible for the inhibition of $\mathrm{AE}$ on cell growth, WB and PCR were used to identify E6/E7 levels showing that AE significantly decreased the mRNA and protein expressions. These results suggested that the apoptosis of cervical cancer cells induced by AE was related to the expression of E6/E7.

Constitutive glucose uptake is a hallmark of cancer cells and provides energy and biosynthetic material for cancer cell proliferation. ${ }^{24}$ Malignant cells accelerated metabolism and needed more ATP production. It was first observed by Warburg $^{25}$ that the rate of aerobic glycolysis was increased in cancer cells, which resulted in the accumulation of lactate due to the elevated levels of lactate dehydrogenase converting pyruvate to lactate. ${ }^{26}$ Moreover, other studies have proposed that tumors use lactate as a fuel, expanding its metabolic functions in cancer. ${ }^{27}$ We supposed that $\mathrm{AE}$ has a suppression on cervical cancer cells by adjusting glucose metabolism. Thus, glucose uptake, lactate production and ATP production were measured in Hela and SiHa cells after treated with $\mathrm{AE}$ at doses of 50 and $100 \mu \mathrm{M}$, and the result showed that $\mathrm{AE}$ indeed inhibited glucose metabolism in a concentration and time-dependent manner. 
A

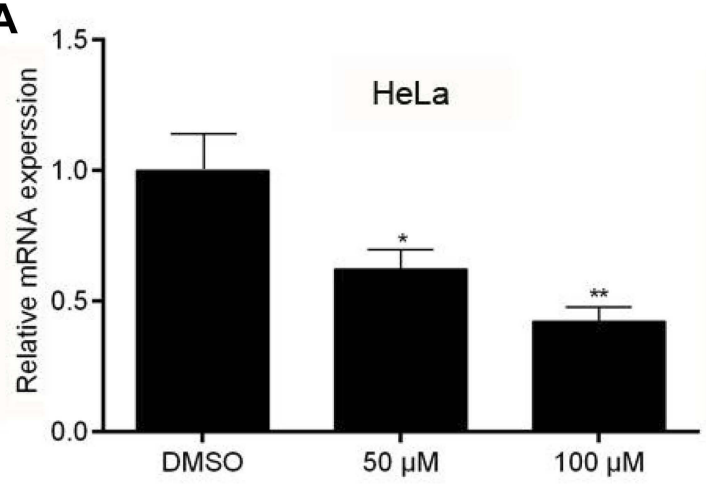

C
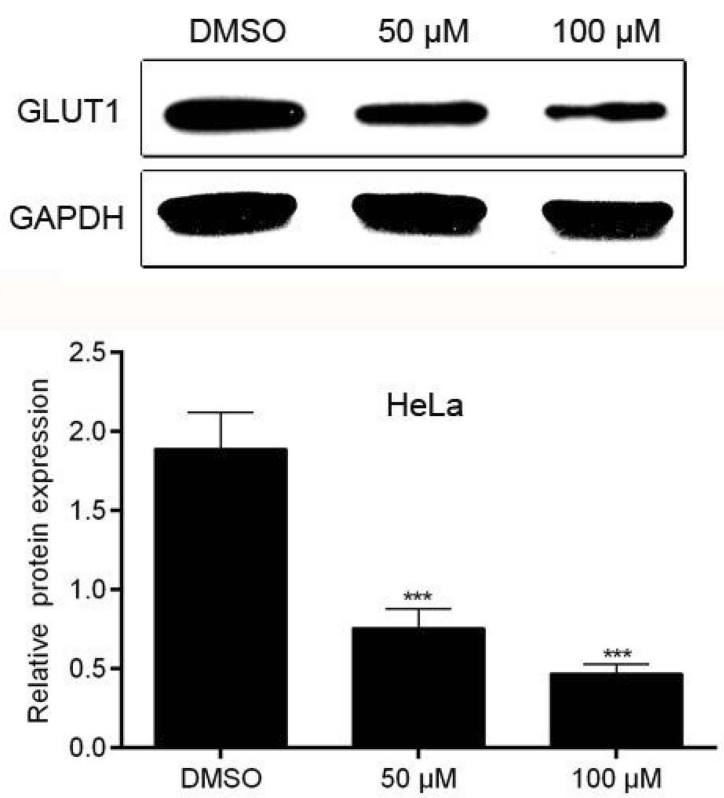

B

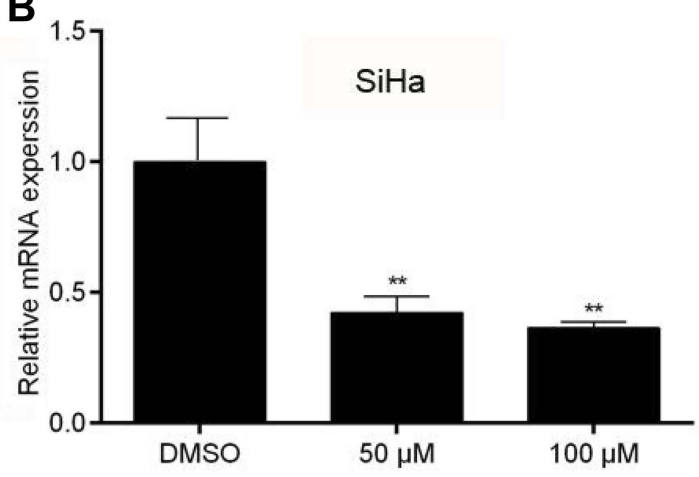

D $\quad \mathrm{SiHa}$
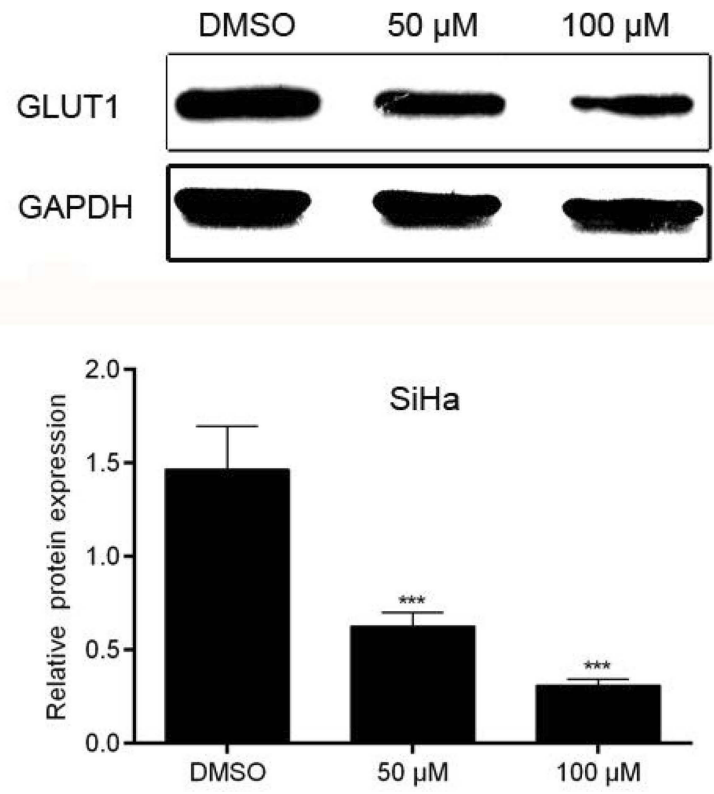

Figure 4 Effect of AE on the expressions of GLUTI in cervical cancer cells. (A-B) The relative mRNA expression of GLUTI was measured by qRT-PCR in Hela and SiHa cells. (C-D) The relative protein expression of GLUTI was tested by WB, showing a significant depression just like the expression of its mRNA in Hela and SiHa cells. $*_{p}<0.05, * * p<0.01$ and $* * * p<0.001$ compared with the data from the respective control group detected at same time point.

We hypothesized that the inhibitory effect of AE on tumor metabolism was achieved by adjusting the level of GLUT 1. To define whether the restrained metabolism is associated with GLUT1, the expressions of GLUT1 and relative mRNA were measured in Hela and $\mathrm{SiHa}$ cells treated with AE. The results showed that the protein level and mRNA level of GLUT1 have a negative correlation with $\mathrm{AE}$ in a concentration-dependent manner. In other words, AE inhibits the expression of GLUT1.

To further verify the role of GLUT1 in the anti-tumor effect of AE, we overexpressed GLUT1 in Hela cells. As expected, overexpression of GLUT1 not only weakened the inhibitory effects of $\mathrm{AE}$ on glucose uptake, lactic production and ATP production, but also decreased the cell apoptosis induced by AE. Taken together, these data demonstrated that $\mathrm{AE}$ played an anti-tumor effect through inhibiting glucose metabolism.

\section{Conclusion}

In this study, we found that the anti-tumor effect of $\mathrm{AE}$ in cervical cancer cells was associated with HPV E6/E7 and glucose metabolism. Research has been reported that overexpressed E6/E7 increased the protein and mRNA levels of GLUT1 in lung cancer cells. ${ }^{28}$ So, we will further explore the relationship between E6/E7 and GLUT1 in the anti-tumor effect of AE in cervical cancer. What's more, the anti-tumor effect of $\mathrm{AE}$ in vivo was also required to further study. 
A

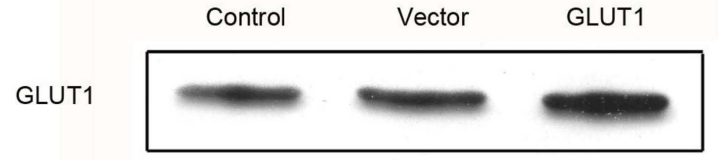

GAPDH

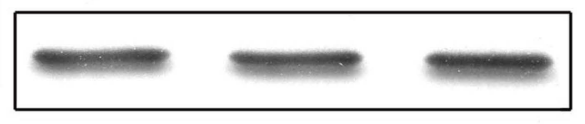

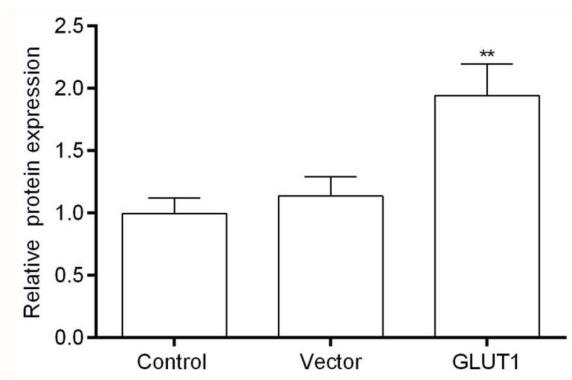

B

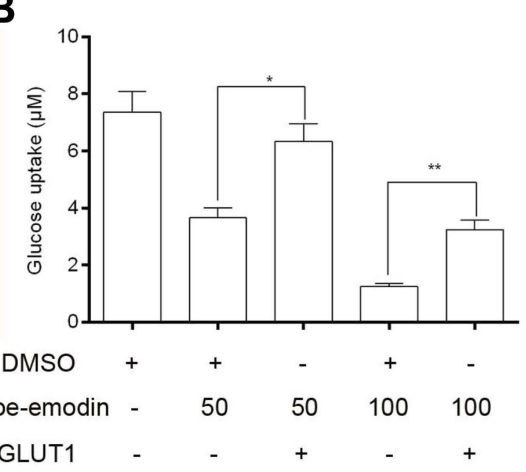

C

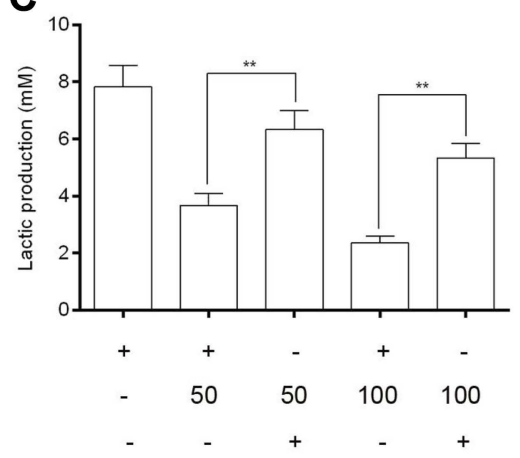

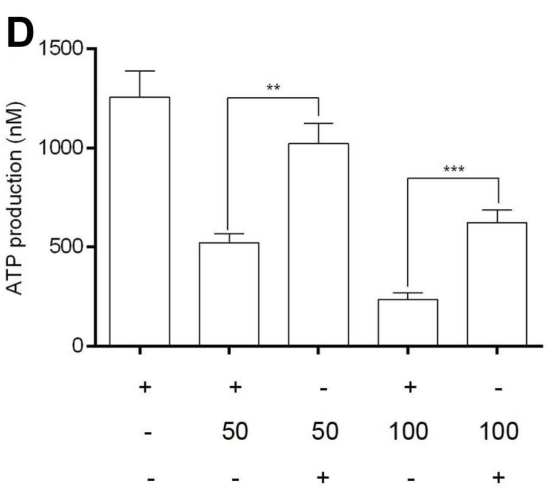

Figure 5 Overexpression of GLUTI weakened the inhibitory effect of AE on glucose metabolism in HeLa cells. (A) The transfection efficiency of pcDNA3.I-GLUTI was evaluated by Western blot. pcDNA3.I vectors were used as negative controls. (B) Overexpression of GLUTI caused an increase in cell glucose uptake. (C) Lactic production was up-regulated after the overexpression of GLUTI. (D) After overexpression of GLUTI, the production of ATP was also increased.* $p<0.05$, $* * 1<0.0$ I and $* * *$ $p<0.00$ I compared with the data from the respective control group detected at same time point.
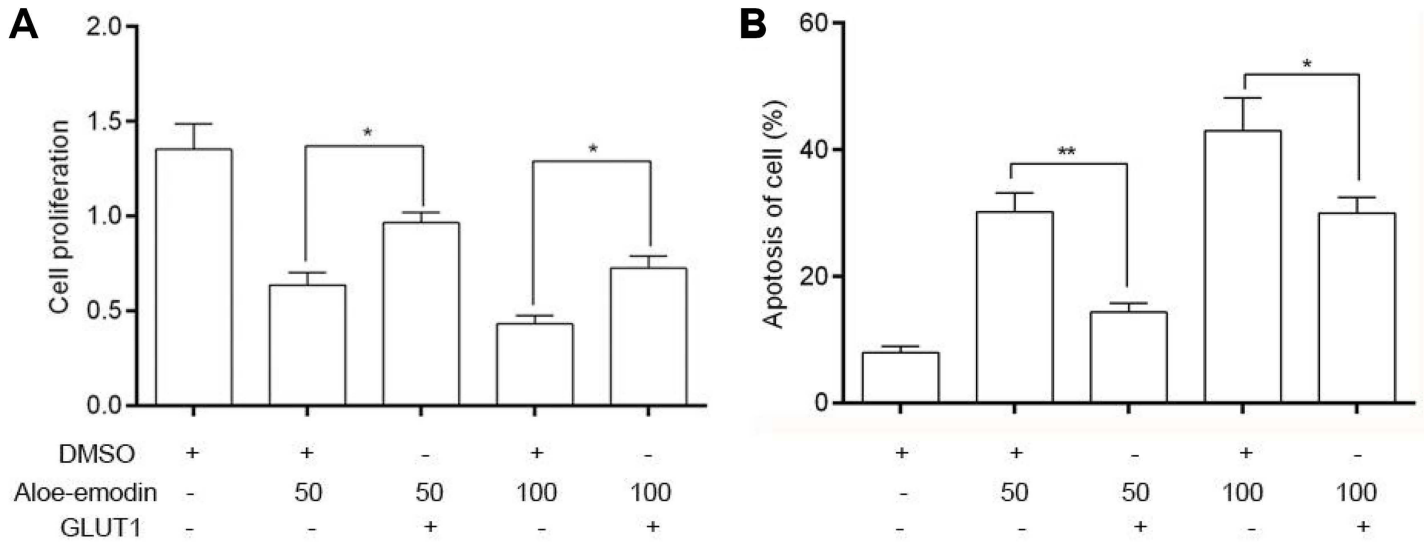

C

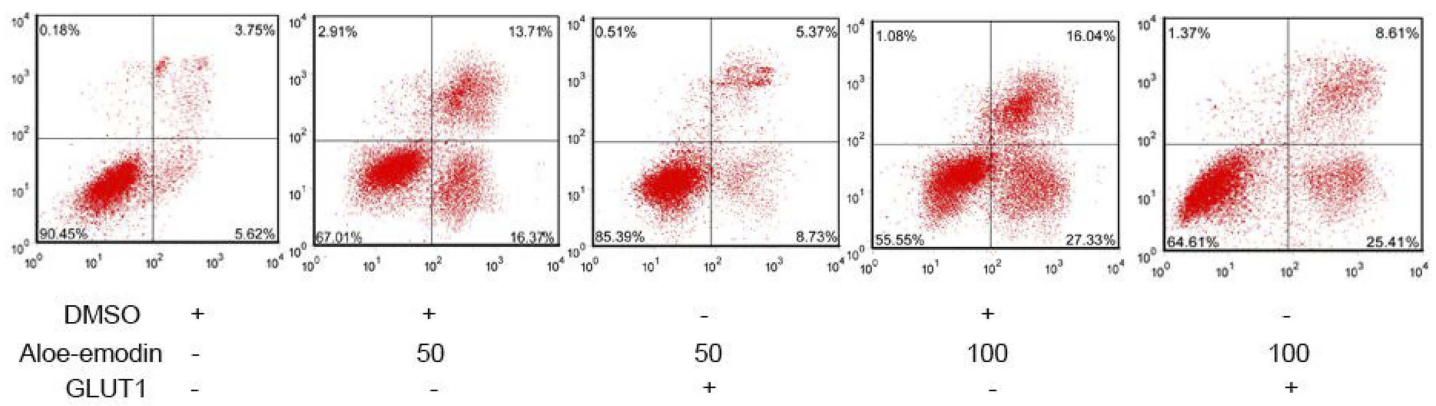

Figure 6 Overexpression of GLUTI inhibited the apoptosis induced by AE in HeLa cells. (A) Overexpression of GLUTI caused a dramatic increase in cell proliferation than untreated cells. (B) Overexpressed GLUTI obviously decreased the apoptosis rate induced by AE. (C) Flow cytometry assay was used to test the apoptosis of HeLa cells. ${ }^{*} p<0.05$ and ${ }^{* *} p<0.01$ compared with the data from the respective control group detected at same time point. 


\section{Acknowledgments}

This study was supported by grants from the Municipal Health Planning Commission's scientific research project (no: [2016] 002) and the Municipal Science and Technology Bureau's scientific research project (no: [2018]1-37).

\section{Disclosure}

The authors report no conflicts of interest in this work.

\section{References}

1. Lin ML, Lu YC, Chung JG, et al. Down-regulation of MMP-2 through the p38 MAPK-NF-kappaB-dependent pathway by aloe-emodin leads to inhibition of nasopharyngeal carcinoma cell invasion. Mol Carcinog. 2010;49(9):783-797. doi:10.1002/mc.20652

2. Xiao BX, Guo J. [The anti-proliferation and anti-migration dual effects of aloe-emodin on KB cells and its mechanism]. Zhonghua Kou Qiang Yi Xue Za Zhi. 2009;44(1):50-52.

3. Jeon W, Jeon YK, Nam MJ. Apoptosis by aloe-emodin is mediated through down-regulation of calpain-2 and ubiquitin-protein ligase E3A in human hepatoma Huh-7 cells. Cell Biol Int. 2012;36 (2):163-167. doi:10.1042/CBI20100723

4. Guo JM, Xiao BX, Liu Q, Zhang S, Liu DH, Gong ZH. Anticancer effect of aloe-emodin on cervical cancer cells involves G2/M arrest and induction of differentiation. Acta Pharmacol Sin. 2007;28 (12):1991-1995. doi:10.1111/j.1745-7254.2007.00707.x

5. Parkin DM, Bray F, Ferlay J, Pisani P. Global cancer statistics, 2002. CA Cancer J Clin. 2005;55(2):74-108.

6. Yoshikawa H. [Progress in the world and challenges in Japan on HPV vaccination for cervical cancer prevention]. Gan To Kagaku Ryoho. 2010;37(6):971-975.

7. Ellenson $\mathrm{LH}, \mathrm{Wu}$ TC. Focus on endometrial and cervical cancer. Cancer Cell. 2004;5(6):533-538. doi:10.1016/j.ccr.2004.05.029

8. Zur Hausen H. Papillomaviruses and cancer: from basic studies to clinical application. Nat Rev Cancer. 2002;2(5):342-350. doi: $10.1038 /$ nrc798

9. Ghittoni R, Accardi R, Hasan U, Gheit T, Sylla B, Tommasino M. The biological properties of E6 and E7 oncoproteins from human papillomaviruses. Virus Genes. 2010;40(1):1-13. doi:10.1007/ s11262-009-0412-8

10. Mesri EA, Feitelson MA, Munger K. Human viral oncogenesis: a cancer hallmarks analysis. Cell Host Microbe. 2014;15 (3):266-282. doi:10.1016/j.chom.2014.02.011

11. McLaughlin-Drubin ME, Munger K. Oncogenic activities of human papillomaviruses. Virus Res. 2009;143(2):195-208. doi:10.1016/j. virusres.2009.06.008
12. Brahimi-Horn C, Pouyssegur J. The role of the hypoxia-inducible factor in tumor metabolism growth and invasion. Bull Cancer. 2006;93(8):E73-E80.

13. Macheda ML, Rogers S, Best JD. Molecular and cellular regulation of glucose transporter (GLUT) proteins in cancer. $J$ Cell Physiol. 2005;202(3):654-662. doi:10.1002/jcp.20166

14. Birnbaum MJ, Haspel HC, Rosen OM. Cloning and characterization of a cDNA encoding the rat brain glucose-transporter protein. Proc Natl Acad Sci U S A. 1986;83(16):5784-5788.

15. Rudlowski C, Becker AJ, Schroder W, Rath W, Buttner R, Moser M. GLUT1 messenger RNA and protein induction relates to the malignant transformation of cervical cancer. Am J Clin Pathol. 2003;120 (5):691-698. doi:10.1309/4KYN-QM58-62JW-2GD7

16. Cantuaria G, Fagotti A, Ferrandina G, et al. GLUT-1 expression in ovarian carcinoma: association with survival and response to chemotherapy. Cancer. 2001;92(5):1144-1150.

17. Higashi K, Ueda Y, Sakurai A, et al. Correlation of Glut-1 glucose transporter expression with [(18)F]FDG uptake in non-small cell lung cancer. Eur J Nucl Med. 2000;27(12):1778-1785. doi:10.1007/ s002590000367

18. Haber RS, Rathan A, Weiser KR, et al. GLUT1 glucose transporter expression in colorectal carcinoma: a marker for poor prognosis. Cancer. 1998;83(1):34-40.

19. Liao H, Wang Z, Deng Z, Ren H, Li X. Curcumin inhibits lung cancer invasion and metastasis by attenuating GLUT1/MT1-MMP/ MMP2 pathway. Int J Clin Exp Med. 2015;8(6):8948-8957.

20. Guo JM, Xiao BX, Dai DJ, Liu Q, Ma HH. Effects of daidzein on estrogen-receptor-positive and negative pancreatic cancer cells in vitro. World J Gastroenterol. 2004;10(6):860-863.

21. Pecere T, Gazzola MV, Mucignat C, et al. Aloe-emodin is a new type of anticancer agent with selective activity against neuroectodermal tumors. Cancer Res. 2000;60(11):2800-2804.

22. Mijatovic S, Maksimovic-Ivanic D, Radovic J, et al. Anti-glioma action of aloe emodin: the role of ERK inhibition. Cell Mol Life Sci. 2005;62(5):589-598. doi:10.1007/s00018-005-4425-8

23. Huang SS, Hao DZ, Zhang Y, Liu HM, Shan WS. Progress in studies of the mechanisms and clinical diagnosis of cervical carcinoma associated with genomic integration of high-risk human papillomavirus DNA. Yi Chuan. 2017;39(9):775-783. doi:10.16288/j.yczz.17-151

24. Hanahan D, Weinberg RA. Hallmarks of cancer: the next generation. Cell. 2011;144(5):646-674. doi:10.1016/j.cell.2011.02.013

25. Warburg O. On the origin of cancer cells. Science. 1956;123 (3191):309-314.

26. Shim H, Dolde C, Lewis BC, et al. c-Myc transactivation of LDH-A: implications for tumor metabolism and growth. Proc Natl Acad Sci U S A. 1997;94(13):6658-6663.

27. Faubert B, Li KY, Cai L, et al. Lactate metabolism in human lung tumors. Cell. 2017;171(2):358-371.e359. doi:10.1016/j.cell.2017.09.019

28. Fan R, Hou WJ, Zhao YJ, et al. Overexpression of HPV16 E6/E7 mediated HIF-1 $\alpha$ upregulation of GLUT1 expression in lung cancer cells. Tumour Biol. 2016;37(4):4655-4663. doi:10.1007/s13277-015-4221-5

\section{Publish your work in this journal}

OncoTargets and Therapy is an international, peer-reviewed, open access journal focusing on the pathological basis of all cancers, potential targets for therapy and treatment protocols employed to improve the management of cancer patients. The journal also focuses on the impact of management programs and new therapeutic agents and protocols on patient perspectives such as quality of life, adherence and satisfaction. The manuscript management system is completely online and includes a very quick and fair peer-review system, which is all easy to use. Visit http://www.dovepress.com/ testimonials.php to read real quotes from published authors. 